\title{
Effects of Pine (Pinus densiflora) Sawdust on Cordycepin Yield from Medicinal Fungus Cordyceps militaris in Submerged Culture
}

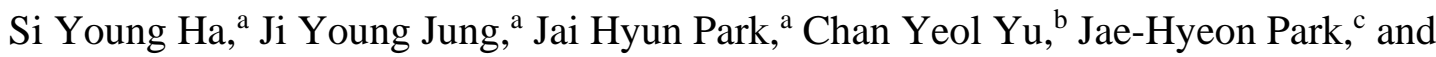
Jae-Kyung Yang ${ }^{\mathrm{a}, *}$

\begin{abstract}
Cordycepin ( $3^{\prime}$-deoxyadenosine) is a nucleoside analog that exhibits a broad spectrum of biological activity. The effects of different tree sawdust on cordycepin as bioactive substances for mycelium growth were investigated. Pine sawdust was essential for increasing cordycepin content. Furthermore, a $1 \% \mathrm{NaOH}$-pretreated pine sawdust produced the highest cordycepin yield. The cordycepin yield of mycelium in submerged culture was significantly increased when the particle size was 100-mesh and the weight was $20 \mathrm{~g} / \mathrm{L}$ of $1 \% \mathrm{NaOH}$-pretreated pine sawdust, with an increase of up to $38 \%$ compared to the control (only sabouraud dextrose broth (SDB)). The results demonstrated the effects of different tree sawdust on the biosynthesis of cordycepin as bioactive substances and that replacing traditional medium (SDB medium) with $1 \% \mathrm{NaOH}-$ pretreated pine sawdust can increase the yield of cordycepin. After optimization of cordycepin production from Cordyceps militaris cultivated in medium containing $1 \% \mathrm{NaOH}$-pretreated pine sawdust using RSM (response surface methodology) BBD (Box-Behnken design) in its canonical form, the optimum combination was: particle size, 113.7-mesh; input weight, $11.9 \mathrm{~g} / \mathrm{L}$; and incubation time, $67.8 \mathrm{~h}$. The model predicted a maximum yield of $922.6 \mu \mathrm{g} / \mathrm{mL}$ for cordycepin.
\end{abstract}

Keywords: Cordyceps militaris; Cordycepin; Wood sawdust; Pine; Response surface methodology

Contact information: a: Division of Environmental Forest Science/Institute of Agriculture and Life Science, Gyeongsang National University, Jinju, 52828, Republic of Korea; b: Gyeongsangnam-do Forest Environment Research Institute, Jinju, 52615, Republic of Korea; c: Department of Forest Resources, College of Life Science, Gyeongsang National University, Jinju, 52828, Republic of Korea;

*Corresponding author: jkyang68@gmail.com

\section{INTRODUCTION}

Cordycepin (3-deoxyadenosine), a nucleoside analog, is the main bioactive ingredient of Cordyceps and is known to mediate a variety of pharmacological effects (Tuli et al. 2014). Many chemically modified cordycepin derivatives exhibit various potential therapeutic effects, including anticancer (Nakamura et al. 2015; Shao et al. 2016; Yoon et al. 2018), anti-inflammatory (Kim et al. 2006), and neuroprotective (Olatunji et al. 2016). Some Cordyceps species have been used for a long time for medicinal purposes in China, Japan, Korea, and other oriental countries due to their biological and pharmacological activities, which are generally attributed to the presence of important bioactive ingredients such as adenosine, cordycepin, and exopolysaccharides (Ling et al. 2002; Kim et al. 2003; Ng and Wang 2005). Artificially produced fruiting bodies were investigated by conducting a sequence analysis of the internal transcribed tracer (ITS) region. The results showed that specimens of the fungus Cordyceps militaris (C. militaris) 
from Britain, China, Japan, Korea, and Norway exhibit an extremely low level of genetic variation that was not correlated with their geographical origin (Cui 2015). In addition, there remains much to learn about the nature and evolution of the interactions of Cordyceps spp., both with their host organisms and with the environment (Zheng et al. 2011). Therefore, it is necessary to study biotechnological production and applications to accelerate the development of physiologically active substances based on Cordyceps spp. (Cui and Zhang 2012). Although cordycepin can be synthesized chemically, the procedure is cumbersome, and it uses a large quantity of harmful organic solvents and requires complicated separation that leads to a low yield. Cordycepin is specifically found in Cordyceps, and because they grow slowly in nature, artificial cultivation using liquid medium is preferred. In the liquid medium, for induction of cordycepin production from Cordyceps mycelium, glucose, sucrose, maltose, and galactose are used as carbon sources (Mao et al. 2005).

The production speed of cordycepin is slow in general medium composition such as glucose; therefore, natural substances are added to the culture to increase the production speed and quantity of cordycepin (Mao and Zhong 2004). A method of culturing mycelium that simultaneously uses both solid and liquid media is being attempted using wooden materials as natural substances, and the use of wooden materials in liquid media is economical by replacing existing carbon sources such as glucose. Different approaches to produce cordycepin from Cordyceps have been reported. Shih et al. (2007) reported that the best nitrogen source for cordycepin production is obtained from a submerged culture of $C$. militaris CCRC32219 with yeast extract, whereas Cui and Zhang (2011) reported that high cordycepin content is obtained in medium containing $\mathrm{Mg}^{2+}$. To further enhance cordycepin production by submerged cultivation of Cordyceps, the effects of carbon sources and carbon/nitrogen ratios were investigated using central composite design and response surface analysis, which resulted in cordycepin production of $345.4 \pm 8.5 \mu \mathrm{g} / \mathrm{mL}$ (Mao et al. 2005). However, low levels of cordycepin are produced in mycelium and culture broth during submerged cultivation of Cordyceps sp.; only $7.1 \mathrm{mg} / \mathrm{L}$ of cordycepin was produced in submerged cultivation of Cordyceps on a laboratory bioreactor scale (Hsu et al. 2002).

Although the above studies aimed for high cordycepin production, none focused on the effects of medium containing sawdust on Cordyceps growth and cordycepin production in a submerged culture. According to the research of Lee et al. (2008), by adding wood powder to the mycelium liquid culture, the mycelium promoted the production of extracellular polysaccharides. Furthermore, it enhanced the production and physiological activity of the produced polysaccharide. Ma et al. (2016) reported that the liquid spawn of Sparassis latifolia led to quick mycelial growth in a pine sawdust substrate compared to in a sawdust spawn. Studies on the effect of wood flour liquid medium on cordycepin enhancement of Cordyceps sinensis are insufficient; therefore, studies to maximize cordycepin induction content from woody liquid medium supplemented with various wood flour are needed. According to Lee et al. (2008), the residue after alkali extraction of the pine tree reported that some phenolic substances, including lignin, were removed, which is beneficial for mushroom mycelium growth. Therefore, it is hypothesized that chemically pretreated wood flour might increase the production of cordycepin in the Cordyceps mycelium, and specific studies on this are required.

Response surface methodology (RSM), a powerful statistical and mathematical tool, helps to identify the effective factors, study the interactions, select optimal conditions, and quantify the relationships between one or more measured responses and the vital input 
factors for a limited number of experiments (Zhang et al. 2010; Cui 2010). This method has been applied successfully in many areas of biotechnology, such as the optimization of nutritional conditions (Cui and Zhang 2011; Cui et al. 2016).

In this study, wood sawdust of pine and pretreated sawdust $(1 \% \mathrm{NaOH}$, water, and ethanol) were used during the submerged culture of $C$. militaris to investigate the effects of pine sawdust on the content of cordycepin. In addition, the culture conditions were optimized, including particle size of pretreated pine sawdust, input weight of pretreated pine sawdust, and incubation time in medium containing pretreated pine sawdust, using RSM based on Box-Behnken design (BBD) to maximize cordycepin content.

\section{EXPERIMENTAL}

\section{Preliminary Experiments}

Preparation of wood sawdust

Four species of plants were used in the experiments for producing wood sawdust: pine (Pinus densiflora), Japanese cedar (Cryptomeria japonica), oak (Quercus variabilis Blume), and tulip tree (Liriodendron tulipifera) (Fig. 1).

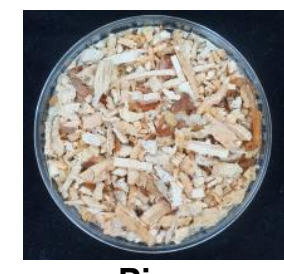

Pine (Pinus densiflora)

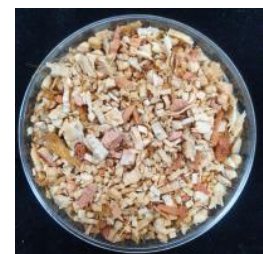

Japanese cedar (Cryptomeria japonica)

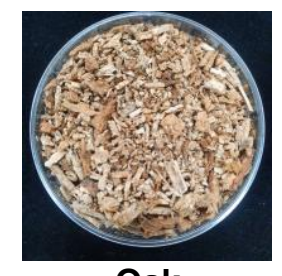

Oak

(Quercus variabilis Blume)

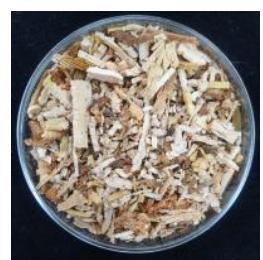

Tulip tree

(Liriodendron tulipifera)

Fig. 1. Wood sawdust samples used for submerged culture of $C$. militaris

These tree species were chosen because of their abundance in South Korea and the fact that they are not typically used in submerged cultures for mycelial growth. Samples of the four tree species were obtained from the Gyeongsang National University Research Forest, Jinju, South Korea. The fresh trunk (including the bark) of each tree species was ground into sawdust using a circular saw (YM-450C, Yulim Co., Ltd., South Korea). The sawdust was then sieved using different meshes to obtain particles with approximate diameters of 106,125 , and $150 \mu \mathrm{m}$.

Table 1. Testing Methods Used

\begin{tabular}{|c|c|c|}
\hline Composition & Methods & Description \\
\hline Carbohydrates & ASTM E1821-96 (1998) & $\begin{array}{c}\text { Standard test method for determination of } \\
\text { carbohydrates in biomass by gas chromatography }\end{array}$ \\
\hline Lignin & Sluiter et al. (2008) & $\begin{array}{c}\text { Determination of structural carbohydrates and } \\
\text { lignin in biomass }\end{array}$ \\
\hline Extractives & Sluiter et al. (2005) & Determination of extractives in biomass \\
\hline Ash & $\begin{array}{c}\text { Sluiter } \text { et al. } \\
(2005)\end{array}$ & Determination ash in biomass \\
\hline
\end{tabular}


Table 2. Chemical Composition (\%, Dry Weight) of Wood Sawdust

\begin{tabular}{|c|c|c|c|c|c|c|c|c|}
\hline $\begin{array}{c}\text { Saw- } \\
\text { dust }\end{array}$ & Arabinose & Xylose & Mannose & Galactose & Glucose & Lignin & $\begin{array}{c}\text { Extrac- } \\
\text { tives }\end{array}$ & Ash \\
\hline Oak & $3.0 \pm 0.0$ & $8.3 \pm 0.0$ & $2.5 \pm 0.0$ & $1.8 \pm 0.0$ & $41.0 \pm 0.0$ & $26.9 \pm 0.2$ & $2.4 \pm 0.0$ & $0.5 \pm 0.0$ \\
\hline Pine & $2.0 \pm 0.0$ & $18.0 \pm 0.0$ & $4.0 \pm 0.0$ & $6.0 \pm 0.0$ & $39.0 \pm 0.0$ & $24.1 \pm 0.0$ & $2.3 \pm 0.0$ & $0.2 \pm 0.0$ \\
\hline Cedar & $2.1 \pm 0.0$ & $15.3 \pm 0.0$ & $3.7 \pm 0.0$ & $5.5 \pm 0.0$ & $40.2 \pm 0.0$ & $25.8 \pm 0.0$ & $2.1 \pm 0.0$ & $0.1 \pm 0.0$ \\
\hline $\begin{array}{c}\text { Tulip } \\
\text { tree }\end{array}$ & $2.7 \pm 0.0$ & $7.7 \pm 0.0$ & $2.4 \pm 0.0$ & $1.7 \pm 0.0$ & $40.9 \pm 0.0$ & $25.1 \pm 0.1$ & $2.5 \pm 0.0$ & $0.5 \pm 0.0$ \\
\hline
\end{tabular}

Table 1 shows the method for measuring the chemical composition of wood sawdust. Table 2 shows the carbohydrates, lignin, extractives, and ash content of the wood sawdust used in the experiment.

\section{Materials}

Effects of the species of plants used for wood sawdust on cordycepin content of $C$. militaris

To study the effect of the species of plants used for wood sawdust on biomass production and cordycepin content of $C$. militaris, various wood sawdust [pine (Pinus densiflora), Japanese cedar (Cryptomeria japonica), oak (Quercus variabilis Blume), and tulip tree (Liriodendron tulipifera)] were tested individually while keeping the other constituents of the basic medium at a constant level. The $C$. militaris strain used in this study was obtained from the Korean Culture Center of Microorganisms (Seoul, South Korea; donation number: 60304), and it was maintained on potato dextrose agar (PDA) plates and sub-cultured every three months. Initially, $C$. militaris was grown on PDA medium in a petri dish and transferred to the seed culture medium by punching out a $5-\mathrm{mm}$ diameter agar disk from the PDA plates. Three disks were used to inoculate $50 \mathrm{~mL}$ of liquid medium. The effect of the species of plants used for sawdust on submerged culture of $C$. militaris was studied using $20 \mathrm{~g} / \mathrm{L}$ of wood sawdust (100-mesh pass) based on the dry weight. The other culture medium (control) used sabouraud dextrose broth (SDB) medium without wood sawdust. Flasks were incubated at $24 \pm 2{ }^{\circ} \mathrm{C}$ for $5 \mathrm{~d}$ with an agitation rate of $100 \mathrm{rpm}$. All experiments were performed 5 times. When the culture was consumed, samples collected from flasks were centrifuged at $6000 \times \mathrm{g}$ for $10 \mathrm{~min}$, and the supernatant was filtered through a pre-weighed Whatman filter paper No. 2 (Whatman International Ltd., Maidstone, UK). The centrifuged mycelium was washed with excess distilled water and collected by filtration using Whatman filter paper. The mycelium was analyzed (cordycepin content and mycelia dry weight) after freeze-drying to a constant dry weight. The effects of different wood sawdust on the cordycepin content of $C$. militaris were assessed. Unpretreated sawdust was used in this experimental set. Based on the results of this experiment, a set of pretreatment experiments was performed for the species showing the highest cordycepin content.

\section{Methods}

Pretreatment of wood sawdust

Water, $1 \% \mathrm{NaOH}$, and ethanol were used for the pretreatment of wood sawdust (100-mesh). The wood sawdust $(5.0 \mathrm{~g})$ was immersed in $100 \mathrm{~mL}$ of solvent. The suspension was subjected to ultrasonication pretreatment (JAC-4020; KODO Technical Research Co., Ltd., Hwaseong, South Korea; $60{ }^{\circ} \mathrm{C}, 20 \mathrm{kHz}, 250 \mathrm{~W}$ ) for $3 \mathrm{~h}$. After wood sawdust 
pretreatment, the sample was cooled to room temperature, and the solid residues were recovered by filtration using a vacuum pump. The solid residues were thoroughly washed with distilled water to a neutral $\mathrm{pH}$ and then dried at $105 \pm 3{ }^{\circ} \mathrm{C}$ for a minimum of $4 \mathrm{~h}$. After drying, the pretreated sawdust was used for submerged culture of $C$. militaris.

\section{Effect of pretreated wood sawdust for cordycepin content in C. militaris on submerged culture}

It has been reported that pretreated wood sawdust medium enhances mycelial production more than untreated wood sawdust medium during higher fungal fermentation (Lee et al. 2008). Thus, based on the results of the preliminary experiments (see Section "Preliminary Experiments"), selected wood sawdust was used for further optimization of the media after pretreatment. Shake flask culturing, containing $50 \mathrm{~mL}$ SDB medium, was conducted in 100-mL Erlenmeyer flasks with a silicone plug. The $\mathrm{pH}$ of the medium was initially adjusted to 5.6 and followed by sterilization at $121{ }^{\circ} \mathrm{C}$ for $15 \mathrm{~min}$. The effect of pretreated solvent of sawdust on submerged culture of $C$. militaris was studied using 5 to $30 \mathrm{~g} / \mathrm{L}$ pretreated sawdust (100-mesh, 120-mesh, and 140-mesh) based on the dry weight. The other culture medium (control) was SDB medium without the pretreated sawdust. Flasks were incubated at $24 \pm 2{ }^{\circ} \mathrm{C}$ for $7 \mathrm{~d}$ with an agitation rate of $100 \mathrm{rpm}$. All experiments were performed 5 times. When the culture was consumed, samples collected from flasks were centrifuged at $6000 \times \mathrm{g}$ for $10 \mathrm{~min}$, and the supernatant was filtered through a pre-weighed Whatman filter paper No. 2 (Whatman International Ltd., Maidstone, UK). The centrifuged mycelium was washed with excess distilled water and collected by filtration using Whatman filter paper. The mycelium was analyzed (cordycepin content and mycelia dry weight) after freeze-drying to a constant dry weight.

\section{Experimental design using response surface methodology (RSM)}

Box-Behnken design has been successfully applied to optimize culture conditions for submerged cultivation of Cordyceps spp. (Shih et al. 2007). A three-level BBD was employed in the present study, and the optimal conditions were determined through a minimal set of experiments compared with other designs (Dong et al. 2009). Fifteen experiments as per the BBD were conducted to explore the effect of culture conditions on the cordycepin (Y1) contents in the submerged cultivation of $C$. militaris. As shown in Table 3, the three factors chosen for this study were particle size (X1), input weight (X2), and incubation time (X3), with each factor at three levels: high (coded as +1), middle (coded as 0 ), and low (coded as -1 ). The design included three replicates at the center point to provide a measure of process stability and inherent variability. All experiments were performed in duplicates. The BBD experimental results were fitted with a second-order polynomial equation (Eq. 1) using a multiple regression technique,

$$
\mathrm{Y}=\beta_{0}+\sum_{i=1}^{4} \beta_{i} x_{i}+\sum_{i=1}^{4} \beta_{i i} x_{i}^{2}+\sum_{i=1}^{3} \sum_{i<j}^{4} \beta_{i j} x_{i} x_{i j}
$$

where $\mathrm{Y}$ is the predicted response (cordycepin yield in this study in $\mathrm{mg} / \mathrm{L}$ ), $\beta_{0}, \beta_{\mathrm{i}}, \beta_{\mathrm{ii}}$, and $\beta_{\mathrm{ij}}$ are constant coefficients, and $x_{\mathrm{i}}$ and $x_{\mathrm{j}}$ are the coded independent variables or factors.

The quality of fit of the second-order model equation was expressed by the coefficient of the determination of $\mathrm{R}^{2}$, and its statistical significance was determined by an F-test. The significance of the regression coefficients was tested using a t-test. The computer software used was Statistical Analysis System (SAS) Design Expert 11 (StatEase Inc., Minneapolis, MN, USA). 
Table 3. Box-Behnken Experimental Design of Medium Containing Pretreated Sawdust for Cordycepin Content of C. militaris on Submerged Culture

\begin{tabular}{|c|c|c|c|c|c|c|c|}
\hline \multirow{2}{*}{ Run } & \multicolumn{3}{|c|}{$\begin{array}{l}\text { Independent Variables } \\
\text { (Coded) }\end{array}$} & \multicolumn{3}{|c|}{$\begin{array}{l}\text { Independent Variables } \\
\text { (Actual) }\end{array}$} & \multirow{2}{*}{$\begin{array}{c}\begin{array}{c}\text { Cordycepin } \\
\text { Content }(\mu \mathrm{g} / \mathrm{mL})\end{array} \\
\mathrm{Y}_{1}\end{array}$} \\
\hline & $X_{1}$ & $X_{2}$ & $X_{3}$ & $X_{1}$ & $X_{2}$ & $X_{3}$ & \\
\hline 1 & 0 & -1 & -1 & 120 & 10 & 24 & 595.0 \\
\hline 2 & 0 & 0 & 0 & 120 & 20 & 72 & 755.3 \\
\hline 3 & 0 & 0 & 0 & 120 & 20 & 72 & 801.5 \\
\hline 4 & -1 & 0 & 1 & 100 & 20 & 120 & 399.7 \\
\hline 5 & -1 & 1 & 0 & 100 & 0 & 72 & 653.3 \\
\hline 6 & 0 & 0 & 0 & 120 & 20 & 72 & 791.2 \\
\hline 7 & 1 & 0 & -1 & 140 & 20 & 24 & 375.7 \\
\hline 8 & 0 & 0 & 0 & 120 & 20 & 72 & 788.3 \\
\hline 9 & 1 & -1 & 0 & 140 & 10 & 72 & 536.0 \\
\hline 10 & -1 & 0 & -1 & 100 & 20 & 24 & 493.0 \\
\hline 11 & 0 & 0 & 0 & 120 & 20 & 72 & 763.2 \\
\hline 12 & 0 & -1 & 1 & 120 & 10 & 120 & 501.7 \\
\hline 13 & 0 & 1 & -1 & 120 & 30 & 24 & 595.0 \\
\hline 14 & 1 & 1 & 0 & 140 & 30 & 72 & 536.0 \\
\hline 15 & 1 & 0 & 1 & 140 & 20 & 120 & 282.3 \\
\hline 16 & 0 & 1 & 1 & 120 & 30 & 120 & 501.7 \\
\hline 17 & -1 & -1 & 0 & 100 & 10 & 72 & 653.3 \\
\hline \multirow{2}{*}{\multicolumn{4}{|c|}{ Independent Variables }} & \multicolumn{4}{|c|}{ Levels } \\
\hline & & & & \multicolumn{2}{|c|}{-1} & 0 & 1 \\
\hline \multicolumn{4}{|c|}{$\begin{array}{c}\mathrm{X}_{1} \text { : Pretreated Sawdust Particle Size, } \\
\text { Mesh }\end{array}$} & \multicolumn{2}{|c|}{100} & 120 & 140 \\
\hline \multicolumn{4}{|c|}{$\mathrm{X}_{2}$ : Pretreated Sawdust Input Weight, $g$} & \multicolumn{2}{|c|}{10} & 20 & 30 \\
\hline \multicolumn{4}{|c|}{$\mathrm{X}_{3}$ : Incubation Time, $\mathrm{h}$} & \multicolumn{2}{|c|}{24} & 72 & 120 \\
\hline
\end{tabular}

\section{Analysis}

Determination of cordycepin

Cordycepin content was analyzed using high-performance liquid chromatography (HPLC, YOUNG IN Chromass Co., Ltd., Anyang, South Korea). One gram of dry mycelium was extracted with $250 \mathrm{~mL}$ of $50 \%$ ethanol under ultrasonic cleaner (JAC-4020; KODO Technical Research Co., Ltd., Hwaseong, South Korea; 20 kHz, 250 W) for 60 min. The supernatant was separated by centrifugation at $18,400 \times \mathrm{g}$ for $10 \mathrm{~min}$ and then filtered through a $0.45-\mu \mathrm{m}$ filter. The filtrate was assayed for cordycepin content. To measure cordycepin, an HPLC system was equipped with a Kinetex $5 \mu \mathrm{C} 18$ 100A column (Phenomenex, Torrance, CA, USA). The mobile phase was $85 \% 0.02 \mathrm{M} \mathrm{KH}_{2} \mathrm{PO}_{4}$ and $15 \%$ methanol and the flow rate was set at $1.2 \mathrm{~mL} / \mathrm{min}$. The injection volume was $20 \mu \mathrm{L}$ and the eluent was detected using a UV/visible detector (Shimadzu SPA-20A, Kyoto, Japan) at 260 
$\mathrm{nm}$. Quantification was based on the UV signal response of each standard using the external standard method, and a standard calibration curve $\left(\mathrm{R}^{2}=0.9912\right)$ was prepared using 100 to 500 ppm cordycepin (Sigma-Aldrich Co., St. Louis, MO, USA). The calibration curve and HPLC chromatogram of the cordycepin standard are shown in Fig. 2.
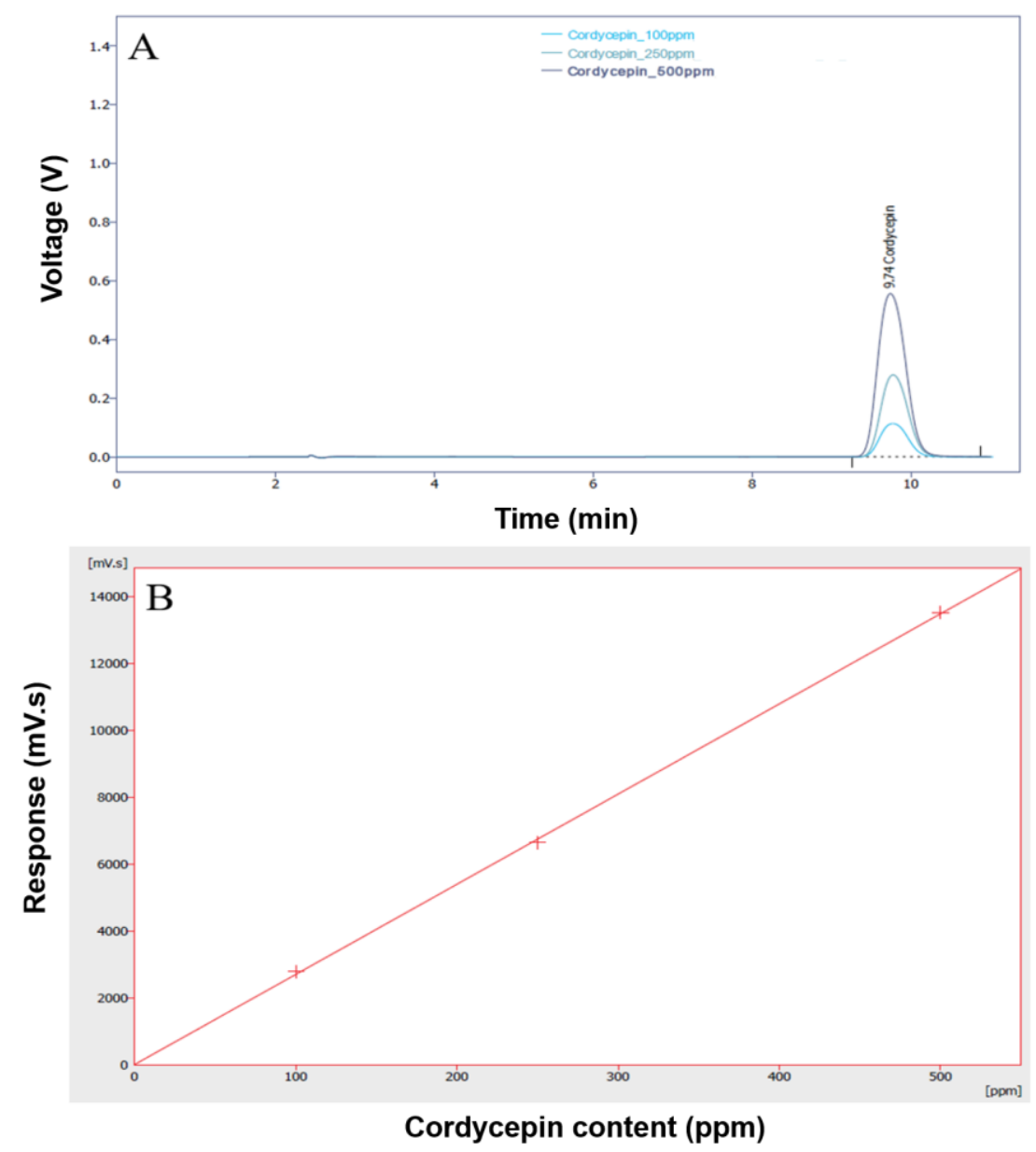

Fig. 2. The HPLC chromatogram and calibration curve of cordycepin standard: A: HPLC chromatogram; B: calibration curve

Determination of mycelium dry weight

Samples collected from flasks were centrifuged at $6000 \times \mathrm{g}$ for $10 \mathrm{~min}$, and the supernatant was filtered through a pre-weighed Whatman filter paper No. 2 (Whatman International Ltd., Maidstone, UK). The centrifuged mycelium was washed with excess distilled water and collected by filtration using Whatman filter paper. The dry weight of the mycelium was measured after freeze-drying to a constant dry weight.

\section{Statistical Analyses}

Data are presented as the mean \pm standard deviation $(n=3)$. Statistical analyses of the results were performed at a 5\% significance level using the Statistical Analysis System software (SAS Institute, Inc., Cary, NC, USA). Differences between the means of individual groups were assessed using SAS with Duncan's multiple-range test. 


\section{RESULTS AND DISCUSSION}

\section{Effect of Various Sawdust Media on Cordycepin Content}

It has been reported that acidic media components are apt for mycelial growth and production of metabolites for many kinds of ascomycetes and basidiomycetes, including Cordyceps sp. (Leung et al. 2006; Liu et al. 2011). The effects of submerged media on the cordycepin content of C. militaris are shown in Fig. 3.

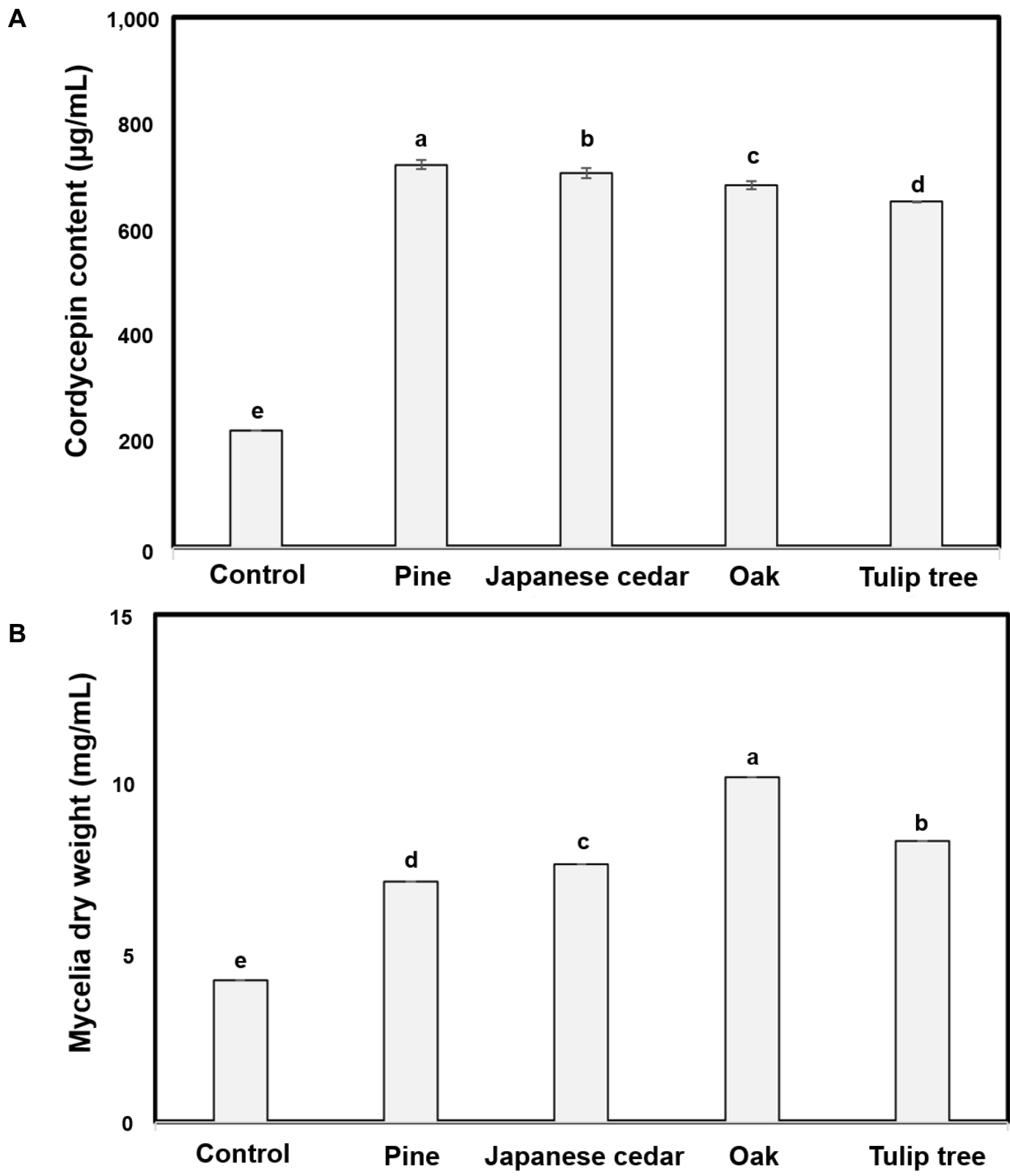

Fig. 3. The effects of various sawdust media on the cordycepin content by $C$. militaris in submerged culture: A: cordycepin content; B: mycelia dry weight; control: only SDB medium; pine (Pinus densiflora), Japanese cedar (Cryptomeria japonica), oak (Quercus variabilis Blume), and tulip tree (Liriodendron tulipifera); incubation time: $5 \mathrm{~d}$. Each value is expressed as mean \pm SE ( $\mathrm{n}$ $=5$ ). Different letters on the top of the line represent statistical significance at $5 \%$ probability level. 
The standard deviation of the results was relatively low, indicating high reproducibility between replicates. In general, there are two methods for quantifying the cordycepin concentration: ultraviolet spectrophotometry and high-performance liquid chromatography (HPLC). Here, we used the HPLC method, which is more sensitive and offers better reproducibility, which may partly explain the high consistency between the replicates. The cordycepin content of $C$. militaris increased slowly after the start of cell growth and reached the maximum of 221.00, 722.49, 706.12, 683.69, and $652.67 \mu \mathrm{g} / \mathrm{mL}$ in the control (only SDB medium), pine (Pinus densiflora), Japanese cedar (Cryptomeria japonica), oak (Quercus variabilis Blume), and tulip tree (Liriodendron tulipifera) sawdust media, respectively. This study also demonstrated that the cordycepin content changed according to the medium containing different wood sawdust. The medium in which wood sawdust was added exhibited a higher content of cordycepin than the control. It should be noted that a direct comparison of metabolite production of various Cordyceps strains from various studies was difficult because the nutrient components and culture conditions used were not the same. It can be seen that the maximum mycelial dry weight for the control (only SDB medium), pine ( $P$. densiflora), Japanese cedar $(C$. japonica), oak $(Q$. variabilis Blume), and tulip tree (L. tulipifera) media was $4.2 \mathrm{~g} / \mathrm{L}, 7.1 \mathrm{~g} / \mathrm{L}, 7.6 \mathrm{~g} / \mathrm{L}, 10.2 \mathrm{~g} / \mathrm{L}$, and 8.3 $\mathrm{g} / \mathrm{L}$ obtained on day 5 , respectively. The authors selected pine sawdust medium with the highest cordycepin content of $C$. militaris and proceeded to perform the pretreatment ( $1 \%$ $\mathrm{NaOH}$, water, and ethanol) experiments.

\section{Effect of Pretreatment of Sawdust on Cordycepin Content}

The effects of pretreated pine sawdust on the cordycepin content of $C$. militaris are shown in Fig. 4. When pine sawdust pretreated with $1 \% \mathrm{NaOH}$ was added to the $C$. militaris mycelium, the highest cordycepin content $(880 \mu \mathrm{g} / \mathrm{mL})$ was observed. Pine sawdust pretreated with $1 \% \mathrm{NaOH}$ demonstrated a $124 \%$ increase in cordycepin content when compared to untreated pine sawdust. It was verified that when the pine tree sawdust was pretreated with $1 \% \mathrm{NaOH}$ and the residue was added to the $C$. militaris liquid medium, the mycelium mass was higher than when the pine tree powder was pretreated with water and ethanol. According to Lee et al. (2008), the residue after alkali extraction of the pine tree indicated that some phenolic substances, including lignin, were removed, which is beneficial for mushroom mycelial growth. In this study, when pine wood sawdust was pretreated with $1 \% \mathrm{NaOH}$ and then added to a liquid medium, it led to the highest cordycepin content and mycelia dry weight of $C$. militaris. It was noteworthy in the present study that when pine wood sawdust was pretreated with $1 \% \mathrm{NaOH}$ and added to the liquid medium, cordycepin and mycelium mass of Cordyceps mycelium were simultaneously increased. Generally, cordycepin content and mycelia dry weight in Cordyceps mycelium are inversely proportional (Hung et al. 2009). Therefore, to increase the cordycepin content of $C$. militaris mycelia and increase the mycelium mass simultaneously, it is beneficial to add pine wood sawdust pretreated with $1 \% \mathrm{NaOH}$ to the liquid medium.

Table 4 shows the chemical composition of pine sawdust according to the pretreatment solvent, and it was demonstrated that the lignin content was significantly reduced due to the $1 \% \mathrm{NaOH}$ pretreatment. Therefore, it is hypothesized that the high content of cordycepin in the medium containing pine sawdust pretreated with $1 \% \mathrm{NaOH}$ was because of the reduced lignin content. 
A

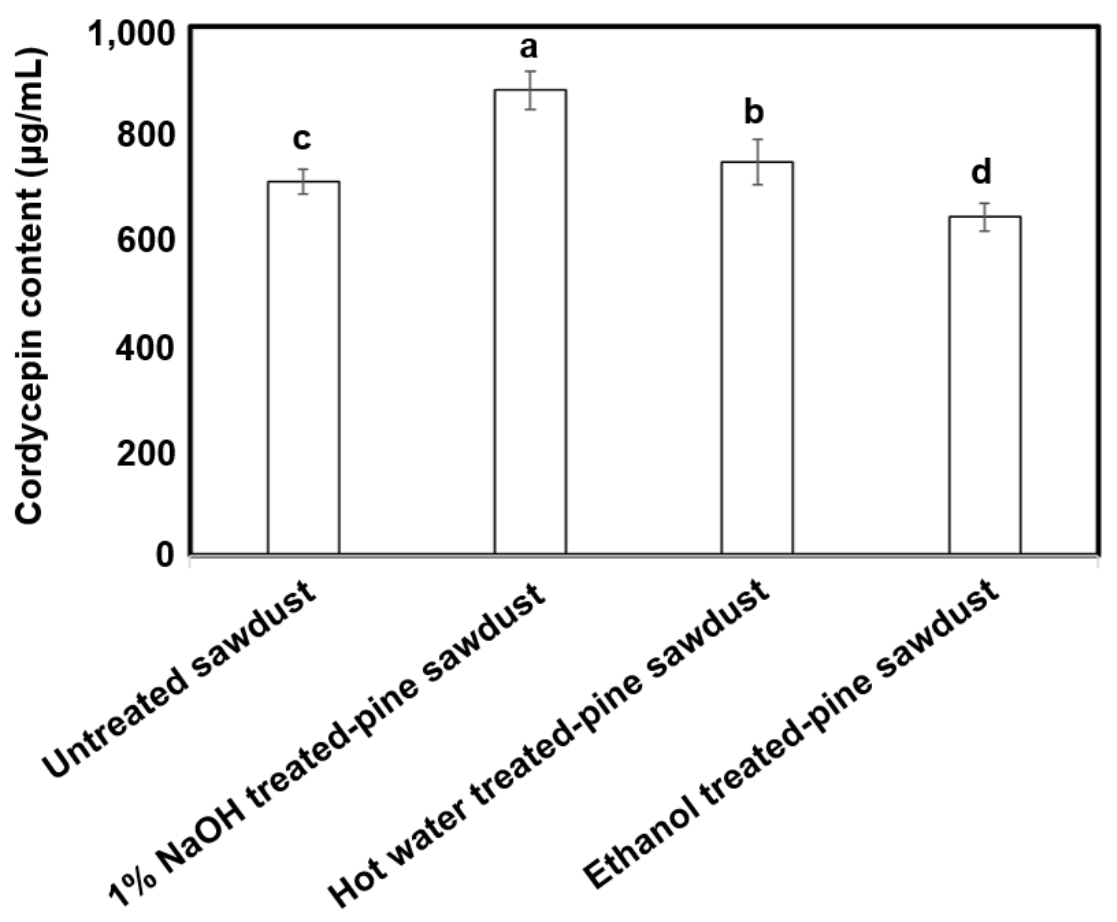

B

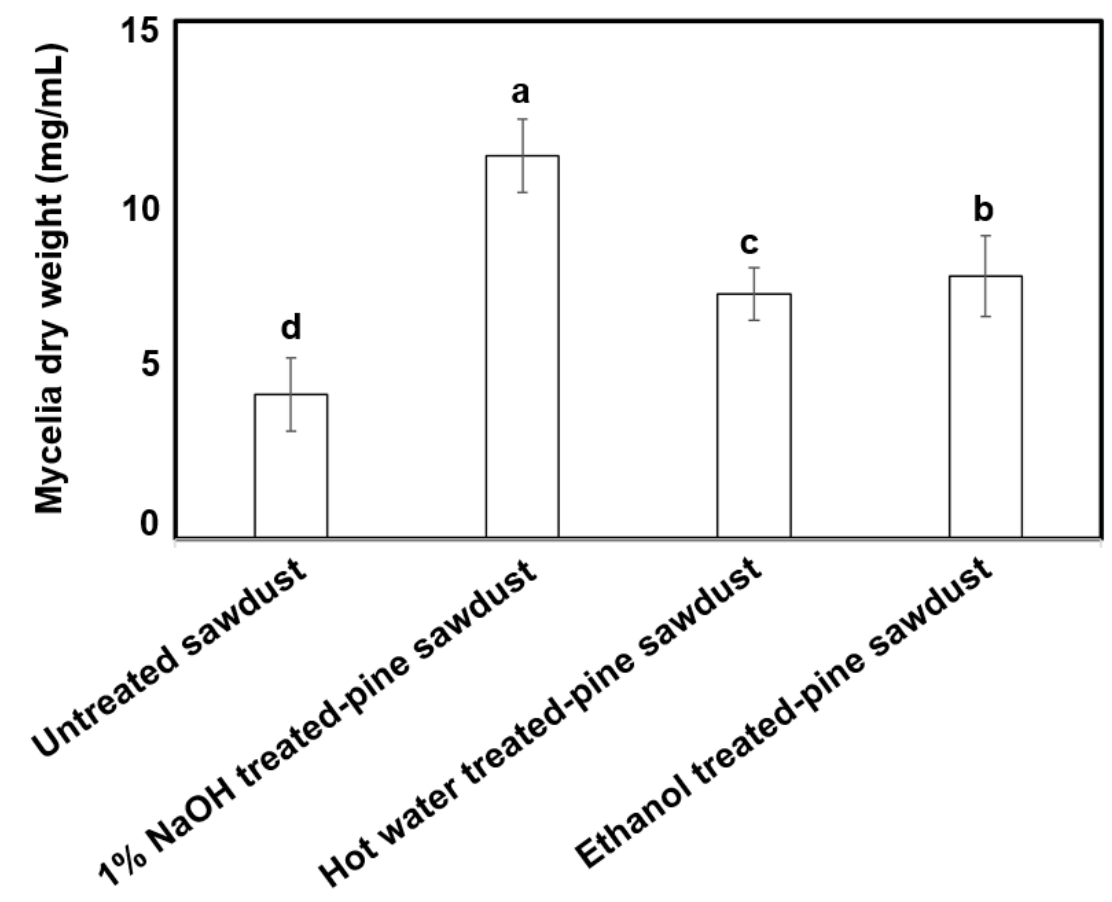

Fig. 4. Effect of pretreatment of pine sawdust (100-mesh, $20 \mathrm{~g} / \mathrm{L}$ ) on cordycepin content in submerged culture of $C$. militaris: A: cordycepin content; B: mycelia dry weight. The $C$. militaris was grown using submerged culture in SDB media; untreated sawdust: untreated pine sawdust; incubation time: $5 \mathrm{~d}$. Each value is expressed as mean $\pm \operatorname{SE}(n=5)$. Different letters on the top of the bars represent statistical significance at $5 \%$ probability level. 
Table 4. Chemical Composition (\%, Dry Weight) of Pretreated Pine Sawdust

\begin{tabular}{|c|c|c|c|c|c|c|c|c|}
\hline $\begin{array}{c}\text { Pretreat- } \\
\text { ment }\end{array}$ & $\begin{array}{c}\text { Arab- } \\
\text { inose }\end{array}$ & Xylose & Mannose & Galactose & Glucose & Lignin & $\begin{array}{c}\text { Extrac- } \\
\text { tives }\end{array}$ & Ash \\
\hline $\begin{array}{c}\text { Non- } \\
\text { treated }\end{array}$ & $2.0 \pm 0.0$ & $18.0 \pm 0.0$ & $4.0 \pm 0.0$ & $6.0 \pm 0.0$ & $39.0 \pm 0.0$ & $24.1 \pm 0.0$ & $2.3 \pm 0.0$ & $0.2 \pm 0.0$ \\
\hline $1 \% \mathrm{NaOH}$ & $1.6 \pm 0.0$ & $36.6 \pm 0.0$ & $2.5 \pm 0.0$ & $4.2 \pm 0.0$ & $40.6 \pm 0.0$ & $10.2 \pm 0.0$ & $5.0 \pm 0.0$ & $5.1 \pm 0.0$ \\
\hline Water & $0.0 \pm 0.0$ & $22.7 \pm 0.0$ & $2.1 \pm 0.0$ & $4.1 \pm 0.0$ & $36.7 \pm 0.0$ & $11.0 \pm 0.0$ & $10.2 \pm 0.0$ & $2.3 \pm 0.0$ \\
\hline Ethanol & $1.5 \pm 0.0$ & $41.8 \pm 0.0$ & $2.0 \pm 0.0$ & $4.1 \pm 0.0$ & $17.2 \pm 0.0$ & $23.1 \pm 0.0$ & $3.0 \pm 0.0$ & $10.2 \pm 0.0$ \\
\hline
\end{tabular}

A
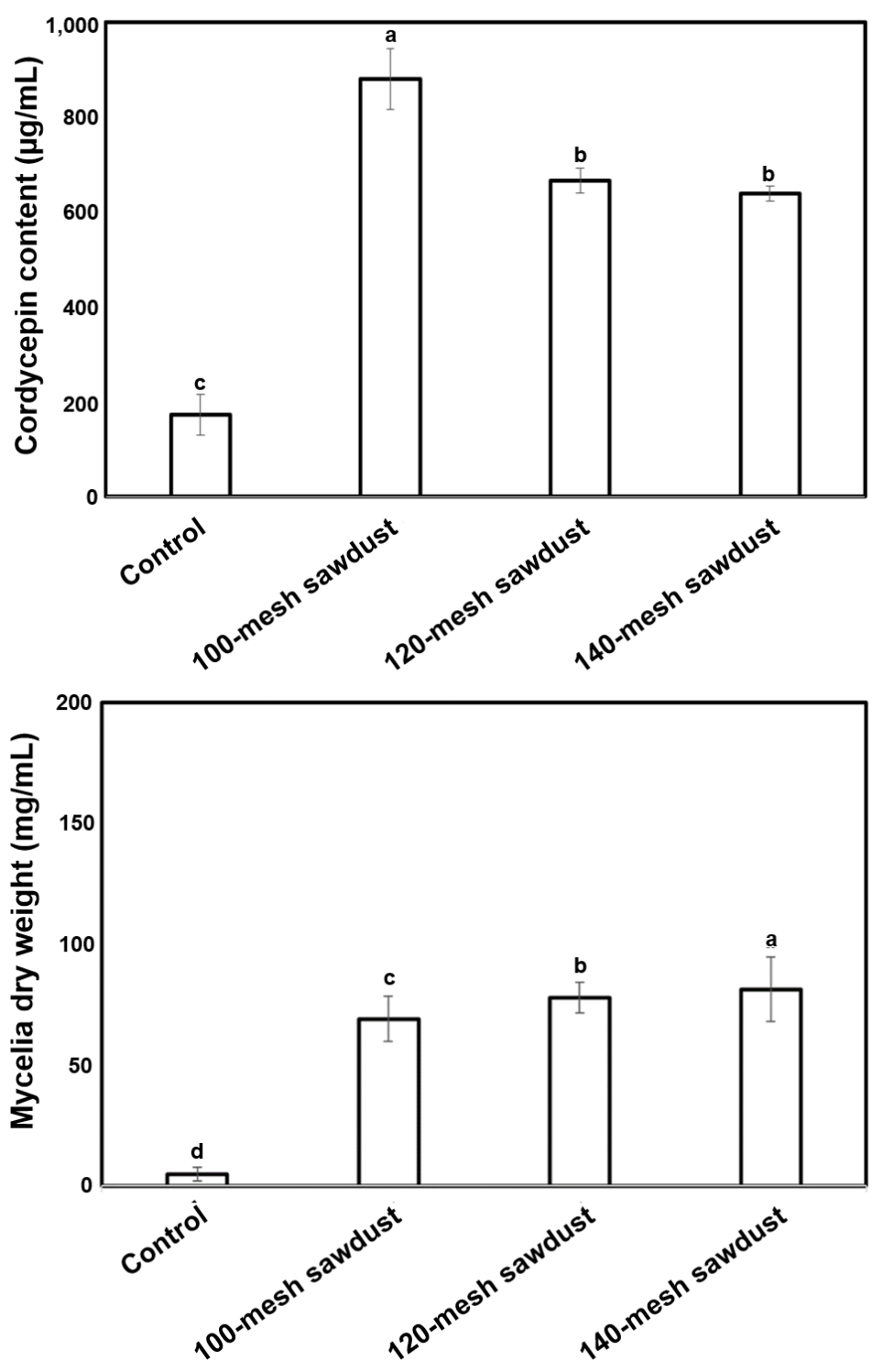

Fig. 5. Effect of particle size of pretreated pine sawdust $(20 \mathrm{~g} / \mathrm{L})$ on cordycepin content in submerged culture of $C$. militaris: A: cordycepin content; B: mycelia dry weight. The $C$. militaris was grown using submerged culture in SDB media; control: only SDB medium. Used pine sawdust was $1 \% \mathrm{NaOH}$-treated pine sawdust; incubation time: $5 \mathrm{~d}$. Each value is expressed as mean $\pm S E(n=5)$. Different letters on the top of the bars represent statistically significant at $5 \%$ probability level. 


\section{Effect of Particle Size and Input Weight of 1\% NaOH-pretreated Pine Sawdust on Cordycepin Content}

The cordycepin content by $C$. militaris reached maximum values of $171.7,880.0$, 666.4 , and $638.4 \mu \mathrm{g} / \mathrm{mL}$ in the control (only SDB medium), 100-mesh pine sawdust, 120mesh pine sawdust, and 140-mesh pine sawdust media, respectively (Fig. 5). The effects of submerged media on the cordycepin content according to the sawdust input weight of $C$. militaris are shown in Fig. 6.

A
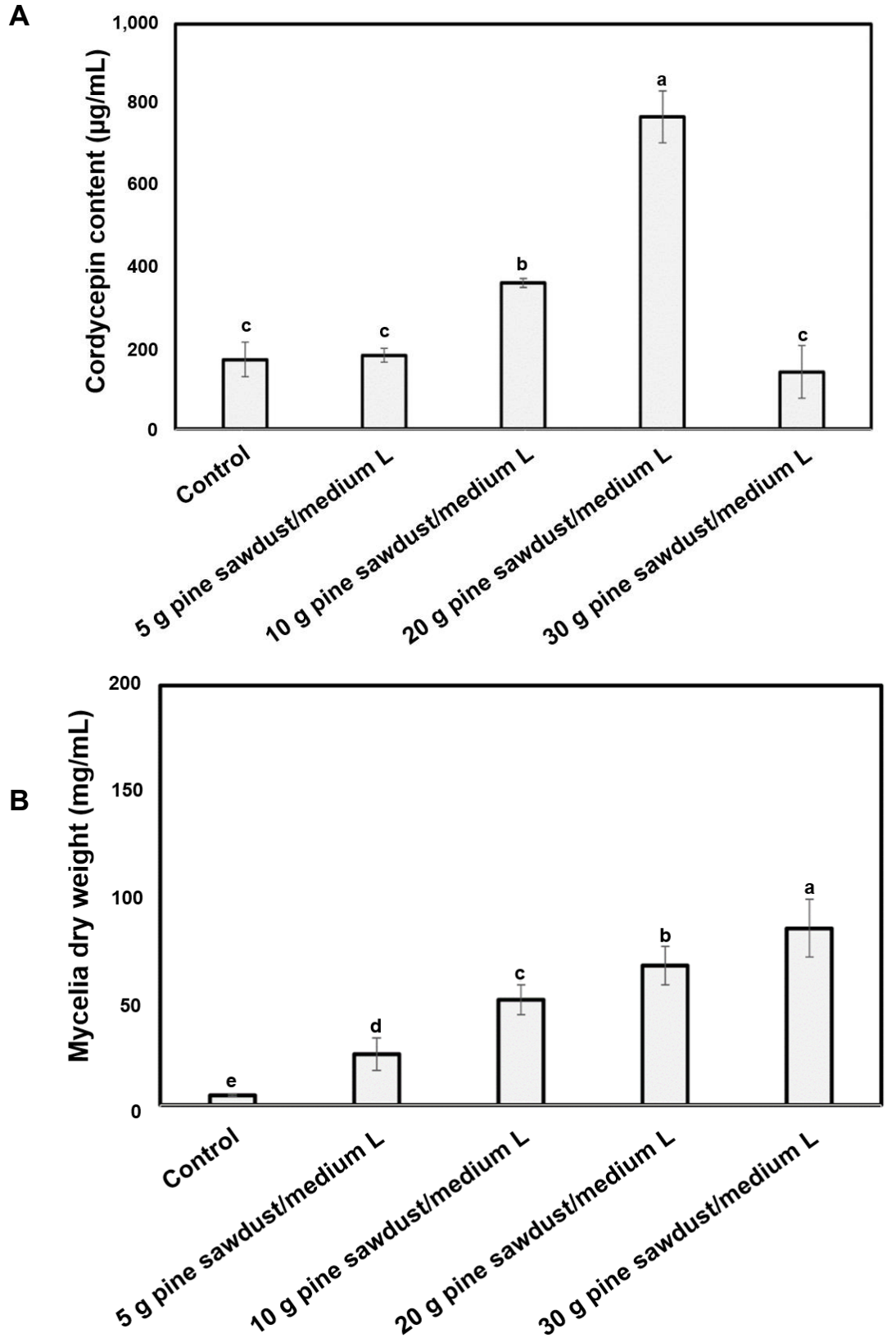

Fig. 6. Effect of particle size of pretreated pine sawdust (100-mesh) on cordycepin content in submerged culture of $C$. militaris: A: cordycepin content; B: mycelia dry weight. The $C$. militaris was grown using submerged culture in SDB media; control: only SDB medium. Used pine sawdust was $1 \% \mathrm{NaOH}$-treated pine sawdust; incubation time: $5 \mathrm{~d}$. Each value is expressed as mean $\pm S E(n=5)$. Different letters on the top of the bars represent statistical significance at $5 \%$ probability level. 
The cordycepin content of $C$. militaris reached a maximum of 171.7, 181.6, 360.4, 880.4 , and $140.5 \mu \mathrm{g} / \mathrm{mL}$ in the control (only SDB medium), $5 \mathrm{~g} / \mathrm{L}, 10 \mathrm{~g} / \mathrm{L}, 20 \mathrm{~g} / \mathrm{L}$, and 30 $\mathrm{g} / \mathrm{L}$ (g: pine sawdust-based dry weight; L: SDB medium-based volume) media, respectively. It should be noted that a direct comparison of metabolite production of various Cordyceps strains from various studies in the literature was difficult because few studies have added wood sawdust into liquid medium. Shukla et al. (2002) demonstrated that the size of sawdust particles greatly influences the adsorption rate. Specifically, decreasing the particle size increases the total surface area, leading to a subsequent increase in the adsorption rate at the sawdust surface. Intraparticle diffusion from the outer surface into the pores of the material is another potentially decisive factor. Thus, the size of sawdust particles can have a significant impact on the cordycepin content. However, additional research is needed to determine the precise relationship between sawdust particle size and cordycepin content.

\section{Incubation Time of $C$. militaris for Cordycepin Production in Medium Containing $1 \% \mathrm{NaOH}$-pretreated Pine Sawdust}

The cordycepin content according to the cultivation time of $C$. militaris is shown in Fig. 7. Cordycepin content rapidly increased after $72 \mathrm{~h}$ of Cordyceps mycelium incubation. The SDB medium was used as a control and the medium containing pine sawdust pretreated with $1 \% \mathrm{NaOH}$ exhibited similar trends. As the incubation time increased, the cordycepin content did not increase significantly; therefore it is essential to find the optimum incubation time for the maximum cordycepin content.

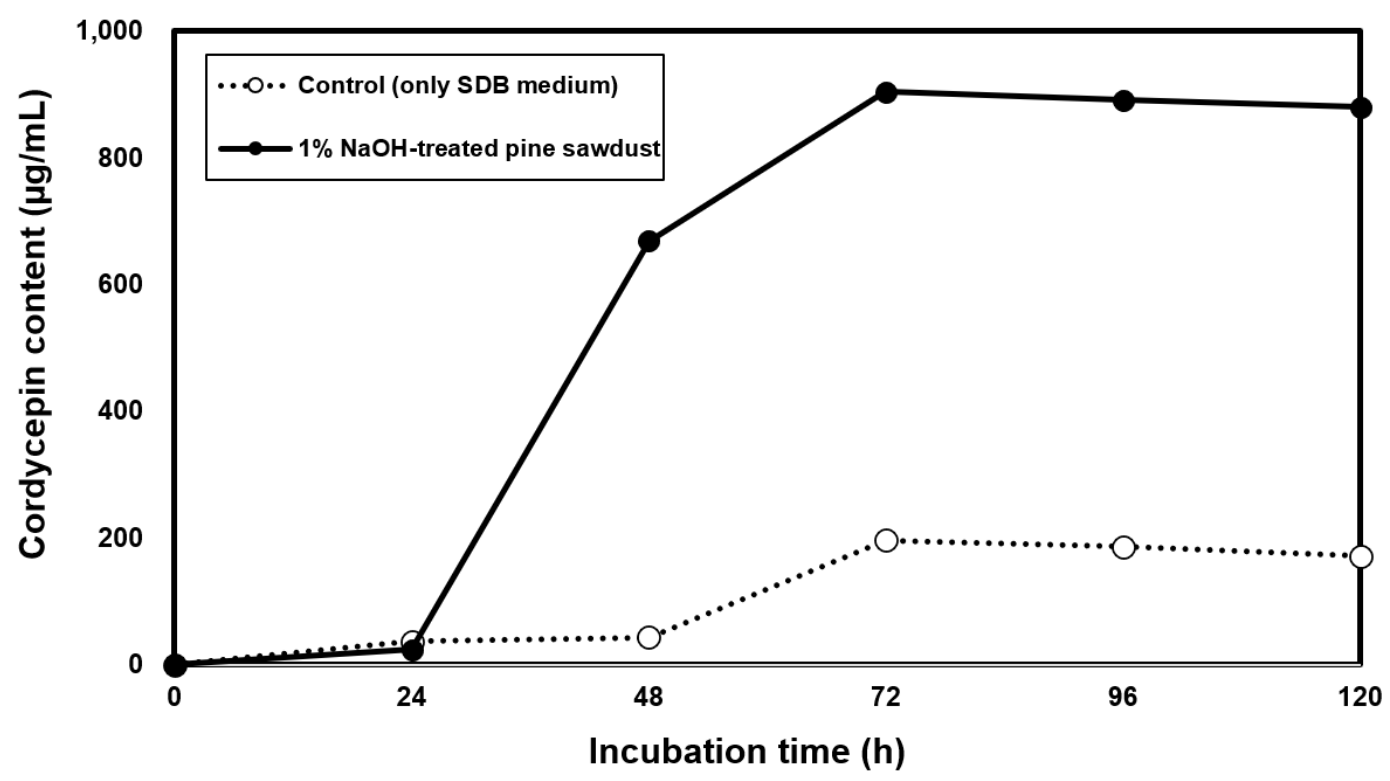

Fig. 7. Effect of incubation time on cordycepin content in submerged culture using medium contained pretreated pine sawdust (100-mesh, $20 \mathrm{~g} / \mathrm{L})$ of $C$. militaris. The C. militaris was grown using submerged culture in SDB media; control: only SDB medium. Used pine sawdust was $1 \%$ $\mathrm{NaOH}$-treated pine sawdust. Each value is expressed as mean $\pm \mathrm{SE}(\mathrm{n}=5)$.

\section{Optimization of Cordycepin Production in C. Militaris Cultivation Medium Containing 1\% NaOH-pretreated Pine Sawdust Using RSM Based on BBD}

Response surface methodology based on BBD was used to determine the optimal input conditions for $1 \% \mathrm{NaOH}$-pretreated pine sawdust in liquid medium. The authors' 
preliminary data indicated that several major variables affect the performance of the culture in terms of cordycepin production: input weight, particle size, and incubation time of the shake flask (Table 1). The matrix corresponding to the BBD is shown in Fig. 8 together with the observed experimental data.
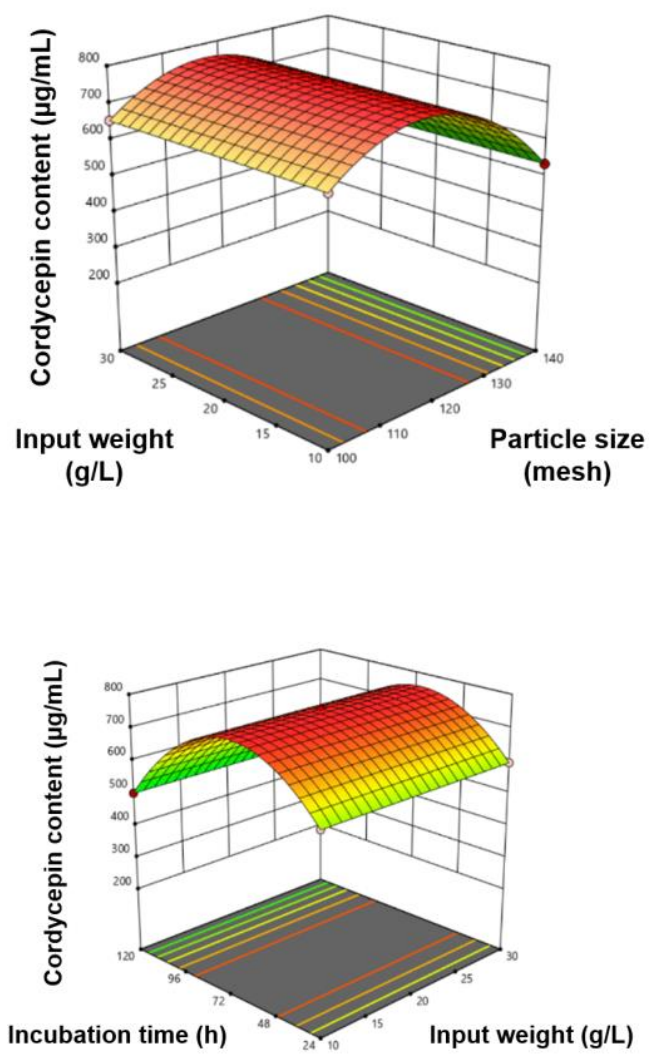
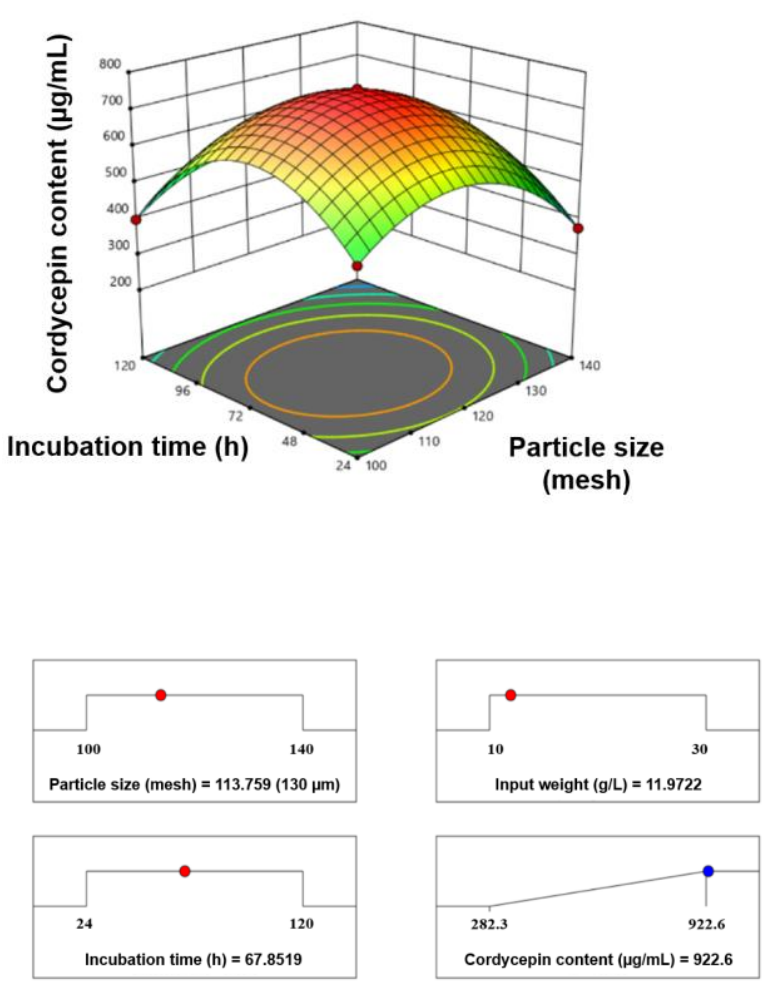

Fig. 8. Regression analysis of the Box-Behnken design experiments

The second-order model equation is given as Eq. 2:

$$
\begin{gathered}
Y_{1}=755.3-58.6625 X_{1}+4.79432 E^{-14} X_{2}-46.6625 X_{3}-5.66269 E^{-14} X_{1} X_{2}- \\
0.025 X_{1} X_{3}+1.62475 E^{-14} X_{2} X_{3}-160.6625 X_{1}^{2}+0.0125 X_{2}^{2}-206.9625 X_{3}^{2}
\end{gathered}
$$

This fit of the model was checked by the coefficient of determination $\mathrm{R}^{2}$, which was calculated as 0.984 , indicating that $98.4 \%$ of the variability in the response could be explained by this model. The $\mathrm{F}$ values for the overall regression were significant at the upper 5\% level, which further supports that the second-order model is adequate for approximating the RSM of the experimental design.

After transforming Eq. 2 to its canonical form, the optimum combination was the following: $1 \% \mathrm{NaOH}$-pretreated pine sawdust particle size, 113.7-mesh $(130 \mu \mathrm{m}) ; 1 \%$ $\mathrm{NaOH}$-pretreated pine sawdust input weight, $11.9 \mathrm{~g} / \mathrm{L}$; and incubation time, $67.8 \mathrm{~h}$. The model predicted a maximum yield of $922.6 \mu \mathrm{g} / \mathrm{mL}$ for cordycepin. The calculated maximum was verified with experiments that were performed in the culture media representing the optimum combination found and a cordycepin yield of $951.9 \mu \mathrm{g} / \mathrm{mL}$ (average of three replicates) was obtained. Although the measured value did not perfectly agree with the value predicted by the response model, it was close to the predicted value. 
Until recently, the levels of cordycepin produced in the mycelium and culture broth during the submerged cultivation of Cordyceps spp. were very low; for example, $7.1 \mu \mathrm{g} / \mathrm{mL}$ of cordycepin was reported for the submerged cultivation of Cordyceps on a laboratory bioreactor scale (Hsu et al. 2002). Following the development of a submerged culture method involving a two-stage dissolved oxygen control for cultivating $C$. militaris for cordycepin production on a commercial scale, Mao and Zhong (2004) achieved a cordycepin level of $188.3 \mu \mathrm{g} / \mathrm{mL}$. To enhance the production of cordycepin via the submerged cultivation of $C$. militaris, the effects of using carbon sources to manipulate the carbon/nitrogen ratio were investigated using central composite design and response surface analysis, resulting in the cordycepin production being elevated to $345.4 \pm 8.5$ $\mu \mathrm{g} / \mathrm{mL}$ (Mao et al. 2005). Prior to this study, the maximum cordycepin level reported (640 $\mu \mathrm{g} / \mathrm{mL}$ ) was found in a surface culture using C. militaris NBRC 9787 (Masuda et al. 2006). The peak cordycepin level obtained in this study $(951.9 \mu \mathrm{g} / \mathrm{mL})$ represents a significant improvement in the values reported in the literature.

\section{CONCLUSIONS}

1. The amount of cordycepin obtained using sawdust was higher than that of the control (only SDB medium), and pine sawdust was the most beneficial for the high content of cordycepin.

2. When pine sawdust was pretreated with $1 \% \mathrm{NaOH}$, water, and ethanol and the residue was applied to a liquid medium, it was found that replacing the traditional medium (SDB medium) with $1 \% \mathrm{NaOH}$-pretreated pine sawdust improved the content of cordycepin.

3. For $1 \% \mathrm{NaOH}$-pretreated pine sawdust, 100-mesh particle size, $20 \mathrm{~g} / \mathrm{L}$ input weight, and $72 \mathrm{~h}$ incubation time were the most effective input conditions for high cordycepin yield.

4. The results of this study suggested that pine sawdust was the most beneficial for enhancing cordycepin content of $C$. militaris in submerged culture.

5. The optimum conditions for cordycepin production were the following: particle size, 113.7-mesh; input weight, $11.9 \mathrm{~g} / \mathrm{L}$; and incubation time, $67.8 \mathrm{~h}$. The model predicted a maximum yield of $922.6 \mu \mathrm{g} / \mathrm{mL}$ for cordycepin.

6. The application of sawdust is a potential strategy for improving the yield of different and useful secondary metabolites from $C$. militaris using submerged culture. However, the mechanisms by which sawdust regulates the metabolic pathways of Cordyceps deserves further investigation.

\section{ACKNOWLEDGMENTS}

The authors are grateful for the support of the Forest Environment Research Institute, Gyeongsangnam-do, South Korea. Additionally, this study was conducted with the support of the 'R\&D Program for Forest Science Technology (Project No. 2019132B1019190001)' provided by Korea Forest Service (Korea Forestry Promotion Institute). 


\section{REFERENCES CITED}

ASTM E1821-96 (1998). "Standard test method for determination of carbohydrates in biomass by gas chromatography," ASTM International, West Conshohocken, PA, USA.

Cui, J. D. (2010). “Optimization of medium for phenylalanine ammonia lyase production in E. coli using response surface methodology," Korean Journal of Chemical Engineering 27(1), 174-178. DOI: 10.1007/s11814-009-0234-3

Cui, J. D., and Zhang, B. Z. (2011). "Comparison of culture methods on exopolysaccharide production in the submerged culture of Cordyceps militaris and process optimization," Letters in Applied Microbiology 52(2), 123-128. DOI: 10.1111/j.1472765X.2010.02987.X

Cui, J. D., and Zhang, Y. N. (2012). "Evaluation of metal ions and surfactants effect on cell growth and exopolysaccharide production in two-stage submerged culture of Cordyceps militaris," Applied Biochemistry and Biotechnology 168, 1394-1404. DOI: 10.1007/s12010-012-9865-7

Cui, J. D. (2015). "Biotechnological production and applications of Cordyceps militaris, a valued traditional Chinese medicine," Critical Reviews in Biotechnology 35(4), 475484. DOI: $10.3109 / 07388551.2014 .900604$

Cui, J., Cui, L., Jia, S., Su, Z., and Zhang, S. (2016). "Hybrid cross-linked lipase aggregates with magnetic nanoparticles: A robust and recyclable biocatalysis for the epoxidation of oleic acid," Agricultural and Food Chemistry 64, 7179-7187. DOI: 10.1021/acs.jafc.6b01939

Dong, C. H., Xie, X. Q., Wang, X. L., Zhan, Y., and Yao, Y. J. (2009). “Application of Box-Behnken design in optimisation for polysaccharides extraction from cultured mycelium of Cordyceps sinensis," Food and Bioproducts Processing 87(2), 139-144. DOI: $10.1016 /$ j.fbp.2008.06.004

Hsu, T. H., Shiao, L. H., Hsieh, C., and Chang, D. M. (2002). “A comparison of the chemical composition and bioactive ingredients of the Chinese medicinal mushroom Dong Chong Xia Cao, its counterfeit and mimic, and fermented mycelium of Cordyceps sinensis," Food Chemistry 78(4), 463-469. DOI: 10.1016/S03088146(02)00158-9

Hung, T. L., Keawsompong, S., Hanh, V. T., Sivichai, S., and Hywel-Jones, N. (2009). "Effect of temperature on cordycepin production in Cordyceps militaris," Thai Journal of Agricultural Science 42(4), 219-225.

Kim, H. G., Shrestha, B., Lim, S. Y., Yoon, D. H., Chang, W. C., Shin, D. J., Han, S. K., Park, S. M., Park, J. H., Park, H. I., et al. (2006). "Cordycepin inhibits lipopolysaccharide-induced inflammation by the suppression of NF-KB through Akt and p38 inhibition in RAW 264.7 macrophage cells," European Journal of Pharmacology 545(2-3), 192-199. DOI: 10.1016/j.ejphar.2006.06.047

Kim, S. W., Hwang, H. J., Xu, C. P., Sung, J. M., Choi, J. W., and Yum, J. W. (2003). "Optimization of submerged culture process for the production of biomass and exopolysaccharides by Cordyceps militaris C738," Journal of Applied Microbiology 94(1), 120-126. DOI: 10.1046/j.1365-2672.2003.01754.x

Lee, W. Y., Ahn, J. K., Park, E. J., and Ka, K. H. (2008). "Effect of wood xylem flour in liquid culture on mycelial biomass of Lentinus lepideus and Lentinus edodes," The Korean Journal of Mycology 36(1), 45-50. DOI: 10.4489/KJM.2008.36.1.045 
Leung, P. H., Zhang, Q. X., and Wu, J. Y. (2006). "Mycelium cultivation, chemical composition and antitumour activity of a Tolypocladium sp. fungus isolated from wild Cordyceps sinensis," Journal of Applied Microbiology 101(2), 275-283. DOI:

10.1111/j.1365-2672.2006.02930.X

Ling, J. Y., Sun, Y. J., Zhang, H., Peng, L., and Zhang, C. K. (2002). "Measurement of cordycepin and adenosine in stroma of Cordyceps sp. by capillary zone electrophoresis (CZE)," Journal of Bioscience and Bioengineering 94(4), 371-374. DOI: $10.1016 / \mathrm{S} 1389-1723(02) 80181-5$

Liu, Z., Li, P., Zhao, D., Tang, H., and Guo, J. (2011). "Anti-inflammation effects of Cordyceps sinensis mycelium in focal cerebral ischemic injury rats," Inflammation 34(6), 639-644. DOI: 10.1007/s10753-010-9273-5

Ma, L., Lin, Y. Q., Yang, C., Ying, Z. H., and Jiang, X. L. (2016). "Production of liquid spawn of an edible mushroom, Sparassis latiflolia by submerged fermentation and mycelial growth on pine wood sawdust," Scientia Horticulturae 209, 22-30. DOI: 10.1016/j.scienta.2016.06.001

Mao, X. B., and Zhong, J. J. (2004). "Hyperproduction of cordycepin by two-stage dissolved oxygen control in submerged cultivation of medicinal mushroom Cordyceps militaris in bioreactors," Biotechnology Progress 20(5), 1408-1413. DOI: 10.1021/bp049765r

Mao, X. B., Eksiwong, T., Chauvatcharin, S., and Zhong, J. J. (2005). "Optimization of carbon source and carbon / nitrogen ratio for cordycepin production by submerged cultivation of medicinal mushroom Cordyceps militaris," Process Biochemistry 40(5), 1667-1672. DOI: 10.1016/j.procbio.2004.06.046

Masuda, M., Urabe, E., Sakurai, A., and Sakakibara, M. (2006). "Production of cordycepin by surface culture using the medicinal mushroom Cordyceps militaris," Enzyme and Microbial Technology 39(4), 341-646. DOI:

10.1016/j.enzmictec.2005.11.010

Nakamura, K., Shinozuka, K., and Yoshikawa, N. (2015). “Anticancer and antimetastatic effects of cordycepin, an active component of Cordyceps sinensis," Journal of Pharmacological Sciences 127(1), 53-56. DOI: 10.1016/j.jphs.2014.09.001

Ng, T. B., and Wang, H. X. (2005). "Pharmacological actions of Cordyceps, a prized folk medicine," Journal of Pharmacy and Pharmacology 57(12), 1509-1519. DOI: 10.1211/jpp.57.12.0001

Olatunji, O. J., Feng, Y., Olatunji, O. O., Tang, J., Ouyang, Z., and Su, Z. (2016). "Cordycepin protects PC12 cells against 6-hydroxydopamine induced neurotoxicity via its antioxidant properties," Biomedicine \& Pharmacotherapy 81, 7-14. DOI: 10.1016/j.biopha.2016.03.009

Shao, L. W., Huang, L. H., Yan, S., Jian, J. D., and Ren, S. Y. (2016). “Cordycepin induces apoptosis in human liver cancer HepG2 cells through extrinsic and intrinsic signaling pathways," Oncology Letters 12(2), 995-1000. DOI: 10.3892/ol.2016.4706

Shih, I. L., Tsai, K. L., and Hsieh, C. (2007). "Effects of culture conditions on the mycelial growth and bioactive metabolite production in submerged culture of Cordyceps militaris," Biochemical Engineering Journal 33(3), 193-201. DOI: 10.1016/j.bej.2006.10.019

Shukla, A., Zhang, Y. H., Dubey, P., Margrave, J. L., and Shukla, S. S. (2002). "The role of sawdust in the removal of unwanted materials from water," Journal of Hazardous Materials 95(1-2), 137-152. DOI: 10.1016/S0304-3894(02)00089-4 
Sluiter, A., Hames, B., Ruiz, R., Scarlata, C., Sluiter, J., Templeton, D., and Crocker, D. (2008). Determination of Structural Carbohydrates and Lignin in Biomass (NREL/TP-510-42618), National Renewable Energy Laboratory, Golden, CO, USA.

Sluiter, A., Hames, B., Ruiz, R., Scarlata, C., Sluiter, J., and Templeton, D. (2005). Determination of Ash in Biomass (NREL/TP-510-42622), National Renewable Energy Laboratory, Golden, CO, USA.

Sluiter, A., Ruiz, R., Scarlata, C., Sluiter, J., and Templeton, D. (2005). Determination of Extractives in Biomass (NREL/TP-510-42619), National Renewable Energy Laboratory, Golden, CO, USA.

Tuli, H. S., Sandhu, S. S., and Sharma, A. K. (2014). "Pharmacological and therapeutic potential of Cordyceps with special reference to Cordycepin," 3 Biotech 4, 1-12. DOI: 10.1007/s13205-013-0121-9

Yoon, S. Y., Park, S. J., and Park, Y. J. (2018). "The anticancer properties of Cordycepin and their underlying mechanisms," International Journal of Molecular Sciences 19(10), article no. 3027. DOI: 10.3390/ijms19103027

Zhang, B. Z., Gui, J. D., Zhao, G. X., and Jia, S. R. (2010). "Modeling and optimization of phenylalanine ammonia lyase stabilization in recombinant Escherichia coli for the continuous synthesis of L-phenylalanine on the statistical-based experimental design," Journal of Agricultural and Food Chemistry 58, 2795-2800. DOI: $10.1021 / \mathrm{jf} 9036744$

Zheng, P., Xia, Y., Xiao, G., Xiong, C., Hu, X., Zhang, S., Zheng, H., Huang, Y., Zhou, Y., Wang, S., Zhao, G. P., Liu, X., Leger, R. J. S., and Wang, C. (2011). 'Genome sequence of the insect pathogenic fungus Cordyceps militaris, a valued traditional Chinese medicine," Genome Biology 12(R116), 1-21. DOI: 10.1186/gb-2011-12-11r116

Article submitted: November 27, 2020; Peer review completed: July 17, 2021; Revised version received and accepted: August 4, 2021; Published: August 5, 2021. DOI: 10.15376/biores.16.4.6643-6660 